Mário Borges Rosa'

Edson Perini"

Tânia Azevedo Anacleto'

Hessem Miranda Neiva'

Tânia Bogutchi"I
Fundação Hospitalar do Estado de Minas Gerais. Belo Horizonte, MG, Brasil

"Departamento de Farmácia Social. Faculdade de Farmácia. Universidade Federal de Minas Gerais. Belo Horizonte, MG, Brasil

III Departamento de Estatística. Pontifícia Universidade Católica de Minas Gerais. Belo Horizonte, MG, Brasil

Correspondência | Correspondence: Faculdade de Farmácia - CEMED Universidade Federal de Minas Gerais Av. Antônio Carlos 6627, sala 3111 31270-901 Belo Horizonte, MG, Brasil E-mail: mariobr_ca@yahoo.com

\section{Erros na prescrição hospitalar de medicamentos potencialmente perigosos}

\section{Errors in hospital prescriptions of high-alert medications}

\section{RESUMO}

OBJETIVO: Os erros de medicação são atualmente um problema mundial de saúde pública, sendo os mais sérios os de prescrição. O objetivo do estudo foi analisar a prática da prescrição de medicamentos de alto risco e sua relação com a prevalência de erros de medicação em ambiente hospitalar.

MÉTODOS: Estudo transversal retrospectivo abrangendo 4.026 prescrições com medicamentos potencialmente perigosos. Durante 30 dias de 2001, foram analisadas todas as prescrições recebidas na farmácia de um hospital de referência de Minas Gerais. As prescrições foram analisadas quanto a: legibilidade, nome do paciente, tipo de prescrição, data, caligrafia ou grafia, identificação do prescritor, análise do medicamento e uso de abreviaturas. Os erros de prescrição foram classificados como de redação ou decisão, sendo avaliada a influência do tipo de prescrição na ocorrência de erros.

RESULTADOS: Houve predomínio da prescrição escrita à mão $(45,7 \%)$. Em $47,0 \%$ das prescrições escritas à mão, mistas e pré-digitadas ocorreram erros no nome do paciente, em $33,7 \%$ houve dificuldades na identificação do prescritor e 19,3\% estavam pouco legíveis ou ilegíveis. No total de 7.148 medicamentos de alto risco prescritos, foram observados 3.177 erros, sendo mais freqüente a omissão de informação $(86,5 \%)$. Os erros se concentraram principalmente nos medicamentos heparina, fentanil e midazolam; e os setores de tratamento intensivo e a neurologia apresentaram maior número de erros por prescrição. Observou-se o uso intensivo e sem padronização de abreviaturas. Quando computados todos os tipos de erros, verificou-se 3,3 por prescrição. A prescrição pré-digitada apresentou menor chance de erros do que as mistas ou escritas à mão.

CONCLUSÕES: Os resultados sugerem a necessidade da padronização no processo de prescrição e a eliminação daquelas feitas à mão. $\mathrm{O}$ uso de prescrições pré-digitadas ou editadas poderá diminuir os erros relacionados aos medicamentos potencialmente perigosos.

DESCRITORES: Erros de Medicação. Prescrição de Medicamentos. Medicamentos com Prescrição. Medicamentos de Controle Especial. Estudos Transversais. 


\section{ABSTRACT}

OBJECTIVE: Medication errors are currently a worldwide public health issue and it is one of the most serious prescription errors. The objective of the study was to evaluate the practice of prescribing high-alert medications and its association with the prevalence of medication errors in hospital settings.

METHODS: A retrospective cross-sectional study was conducted including 4,026 prescription order forms of high-alert medications. There were evaluated all prescriptions received at the pharmacy of a reference hospital in the state of Minas Gerais, southeastern Brazil, over a 30-day period in 2001. Prescription were checked for legibility, patient name, type of prescription, date, handwriting or writing, prescriber identification, drug prescribed, and use of abbreviations. Prescription errors were classified as writing or decision errors and how the type of prescription affected the occurrence of errors was assessed.

RESULTS: Most prescriptions were handwritten (45.7\%). In $47.0 \%$ of handwritten, mixed and pre-typed prescriptions had patient name errors; the prescriber name was difficult to identify in $33.7 \%$; $19.3 \%$ of them were hardly legible or illegible. Of a total of 7,148 high-alert drugs prescribed, 3,177 errors were found, and the most frequent one was missing information $(86.5 \%)$. Errors occurred mostly in prescriptions of heparin, phentanyl, and midazolam. Intensive care and neurology units had the highest number of errors per prescription. Non-standard abbreviations were frequent and widespread. Overall it was estimated 3.3 errors per prescription order form. Pre-typed prescriptions were less likely to have errors compared to mixed or handwritten prescriptions.

CONCLUSIONS: The study results show there is a need for standardizing the prescription process and eliminating handwritten prescriptions. The use of pre-typed or edited prescriptions may reduce errors associated to high-alert medications.

\section{DESCRIPTORS: Medication Errors. Prescriptions, Drug. Drugs with Prescription. Drugs of Special Control. Cross-Sectional Studies.}

\section{INTRODUÇÃO}

Os estudos Harvard Medical Practice Study I e II, ${ }^{4,12}$ marcantes e pioneiros na área de segurança do paciente, mostraram que os eventos adversos relacionados à assistência são comuns e inesperadamente altos em hospitais norte-americanos, acarretando danos permanentes e mortes. A partir desses dois estudos, estimou-se que cerca de 98.000 norte-americanos morrem por ano devido a erros associados à assistência à saúde, sendo estes considerados uma das principais causas de mortes nos Estados Unidos. ${ }^{10}$ Os eventos adversos mais freqüentes relacionados aos medicamentos foram registrados no Harvard Medical Practice Study II, ${ }^{12}$ sendo uma parte considerável deles evitável. Nos últimos anos, o incremento considerável no número de estudos em segurança do paciente levou a um maior conhecimento sobre o assunto, confirmando sua importância como problema mundial. Em consonância com este preocupante quadro, a Organização Mundial de Saúde lançou em 2004 o programa Aliança Mundial para a Segurança do Paciente. Trata-se de um programa permanente que conclama todos os países membros a tomarem medidas para assegurar a qualidade da assistência prestada nas unidades de saúde de todo o mundo. ${ }^{23}$

Recentemente, publicação sobre erros de medicação considerou o nível e as conseqüências desses eventos inaceitáveis, e registra que cada paciente internado em hospitais americanos está sujeito a um erro de medicação por dia. ${ }^{2}$ Conforme Barber et $\mathrm{al}^{3}$ (2003), os erros de prescrição são os mais sérios dentre os que ocorrem na utilização de medicamentos.

Muitos paradigmas são confrontados na discussão de erros em instituições de saúde. Os profissionais de saúde normalmente associam falhas nas suas atividades à vergonha, perda de prestígio e medo de punições. De modo geral, o ambiente nas instituições de saúde não é propício para uma discussão franca sobre o assunto, visando à melhoria do sistema como um todo. Essa tendência à negação com conseqüente subnotificação 
dos erros na área da saúde muitas vezes dificulta a avaliação dos eventos, prejudicando o conhecimento sobre eles. ${ }^{21}$ Outro obstáculo encontrado para o estudo e prevenção de erros de medicação é a falta de padronização e a multiplicidade da terminologia utilizada para classificá-los. Esta situação prejudica a comparação entre os estudos sobre o tema e retarda o conhecimento epidemiológico sobre o assunto. ${ }^{24}$

Embora a maioria dos medicamentos possua uma margem terapêutica segura, alguns fármacos têm risco inerente de lesar o paciente quando existe falha no processo de utilização. Esses fármacos são chamados de high-alert medications ou medicamentos de alto risco, aqui denominados de medicamentos potencialmente perigosos (MPP). Os erros que acontecem com esses medicamentos não são os mais rotineiros, mas quando ocorrem, possuem severidade alta e podem levar a lesões permanentes ou serem fatais. ${ }^{5,8}$

O presente estudo teve por objetivo analisar a prática da prescrição de medicamentos de alto risco e sua relação com a prevalência de erros de medicação em ambiente hospitalar.

\section{MÉTODOS}

Realizou-se um estudo transversal com coleta retrospectiva de dados em todas as segundas vias de prescrições contendo um ou mais MPP, emitidas entre 29 de agosto e 27 de setembro de 2001, recebidas na farmácia hospitalar de um hospital de referência localizado no estado de Minas Gerais.

Conforme Cohen et al ${ }^{5}$ (1998) e Federico ${ }^{8}$ (2007), foram considerados MPP: atracúrio ampola com 25 miligramas (mg), cloreto de potássio ampola com 10 mililitros $(\mathrm{mL})$ a $10 \%$, digoxina comprimido com $0,25 \mathrm{mg}$, dobutamina ampola com $250 \mathrm{mg}$, dopamina ampola com $50 \mathrm{mg}$, epinefrina ampola com $1 \mathrm{mg}$, fentanil ampola $1 \mathrm{~mL}$ com $0,5 \mathrm{mg}$ e frasco-ampola $10 \mathrm{~mL}$ com $0,5 \mathrm{mg}$, fosfato ácido de potássio ampola $10 \mathrm{~mL}$ com $2 \mathrm{mEq} / \mathrm{mL}$, gluconato de cálcio ampola $10 \mathrm{~mL}$ a $10 \%$, heparina ampola 0,25 $\mathrm{mL} / 5.000$ unidades e frasco-ampola $5 \mathrm{~mL}$ com 5.000 unidades $/ \mathrm{mL}$, insulina NPH com 100 unidades $/ \mathrm{mL}$ e regular com 100 unidades $/ \mathrm{mL}$, midazolam ampolas com $50 \mathrm{mg}, 5 \mathrm{mg}$ e $15 \mathrm{mg}$, morfina ampola $1 \mathrm{~mL}$ com 10 $\mathrm{mg}$, nalbufina ampola $1 \mathrm{~mL}$ com $10 \mathrm{mg}$, norepinefrina ampola $1 \mathrm{~mL}$ com $1 \mathrm{mg}$, pancurônio ampola $2 \mathrm{~mL}$ com $4 \mathrm{mg}$, petidina ampola $2 \mathrm{~mL}$ com $100 \mathrm{mg}$, suxametônio frasco-ampola com $100 \mathrm{mg}$, tramadol frasco $10 \mathrm{~mL}$ a $100 \mathrm{mg} / \mathrm{mL}$, vecurônio ampola $1 \mathrm{~mL}$ com $4 \mathrm{mg}$, varfarina comprimido com $5 \mathrm{mg}$. A essa lista de $26 \mathrm{MPP}$ foi necessário incluir mais quatro definições para fins de registro no formulário (midazolam, heparina, insulina e fentanil sem concentração, forma farmacêutica ou outro parâmetro), em função de várias situações em que os MPP apareciam prescritos sem parâmetros, impossibilitando sua classificação na lista original.
O formulário foi elaborado contendo as oito variáveis da pesquisa com suas devidas classificações, utilizado na coleta e construção do banco de dados. O preenchimento do formulário foi feito por estagiários, graduandos em farmácia nos últimos períodos e revisados por dois farmacêuticos hospitalares com pós-graduação em farmácia hospitalar. Uma revisão final foi feita por um terceiro farmacêutico hospitalar, com curso de especialização na área e mais de 15 anos de experiência em farmácia hospitalar. Em caso de discordância, discutia-se a classificação até o consenso. Após revisão, discussão e consenso, os formulários eram encaminhados para a digitação.

Foram utilizadas oito variáveis ou grupos de variáveis e duas definições de erros, conforme especificações a seguir:

1. Legibilidade das prescrições - em função de seu alto grau de subjetividade e dependência da experiência do avaliador, foi estabelecido um padrão de avaliação mais homogêneo possível, reduzindo aspectos da subjetividade envolvida no julgamento. Foi classificada em três possibilidades:

a) grafia com boa legibilidade: lida normalmente sem problemas para entendimento da escrita;

b) grafia pouco legível ou duvidosa: levava maior de tempo de leitura, sem certeza de compreensão total de todas as palavras, números, símbolos e abreviaturas; e

c) grafia ilegível: impossível o entendimento da escrita.

2. Nome do paciente - além dos critérios de legibilidade, esta variável foi classificada em: incompleto (omissão de partes do nome, mas sem problemas na legibilidade); ilegível/alterado (nome ou sobrenome do paciente alterado durante o período de observação); ou não identificado (paciente internado sem documentos e não foi identificado por nenhuma pessoa).

3. Tipo de prescrição - classificada em: pré-digitada (as prescrições feitas em computador e impressas); escrita à mão; mista (prescrição parte digitada e parte escrita à mão).

4. Data da prescrição - além da classificação de legibilidade, pode ser incompleta ou omitida.

5. Caligrafia ou grafia - aplicada ao corpo da prescrição, sendo classificada como ilegível quando pelo menos $50 \%$ das palavras que as compunham estavam indecifráveis.

6. Identificação do prescritor - Foi estabelecida como padrão a identificação completa com os seguintes parâmetros: prescrição assinada ou rubricada, com carimbo, nome do prescritor e número do Conselho Regional de Medicina (CRM) ou Odontologia (CRO) 
legíveis. Todas as outras identificações do prescritor que não eram classificadas conforme o padrão foram consideradas incompletas. Na classificação de prescrição anônima, não existia qualquer identificação do prescritor.

\section{Análise dos MPP - Divida em quatro partes:}

a) legibilidade do MPP: legível ou pouco legível/ duvidosa.

b) forma farmacêutica: confrontou-se o prescrito com referência sobre farmacologia de Fuchs \& Wannmacher ${ }^{9}$ (1998) e a bula do medicamento, sendo classificada como correta ou incorreta; incompleta (na prescrição não estava descrita a forma farmacêutica completa, mas sem problemas de legibilidade); pouco legível/duvidosa (a forma farmacêutica prescrita deixava margem a dúvida e interpretação); omissão (prescrição sem a forma farmacêutica do medicamento).

c) concentração e via de administração: critérios iguais aos da forma farmacêutica.

d) intervalo e taxa de infusão: critérios iguais aos da forma farmacêutica, com acréscimo da classificação "não se aplica", porque em algumas situações o intervalo não é usado. Exemplo: infusão contínua de medicamentos.

Todas as classificações utilizadas são excludentes, em todos os parâmetros avaliados.

8. Uso de abreviaturas - foram contadas quantas e quais as abreviaturas estavam presentes em cada prescrição e registradas como erros somente aquelas pouco legíveis ou duvidosas. As legíveis não foram consideradas erros porque não havia padronização de abreviaturas no hospital estudado. As abreviaturas U ou UI significando unidades foram contadas separadamente, pois são consideradas as abreviaturas mais perigosas., ${ }^{1,2,11}$

Os erros de prescrição foram classificados conforme Dean et $\mathrm{al}^{6}$ (2000). Os erros de decisão são relacionados ao conhecimento do prescritor, como erro na dose, prescrição de dois medicamentos com o mesmo fim, de medicamento não indicado para o paciente ou sem considerar insuficiência renal e hepática e outras. Foram considerados erros de decisão as seguintes classificações: forma farmacêutica, concentração, via de administração, intervalo e taxa de infusão errados. Já os erros de redação relacionam-se ao processo de elaboração da prescrição, tais como ilegibilidade, uso de abreviaturas confusas (UI), omissão de forma farmacêutica, concentração, via administração, intervalo, taxa de infusão, erro na unidade do medicamento e outros. Foram classificados como erros de redação: nome do paciente (pouco legível ou duvidoso, incompleto, ilegível, alterado), data (pouco legível ou duvidosa, omissão, ilegível, incompleta), caligrafia (pouco legível e ilegível), identificação do prescritor (todas aquelas que não estavam completas), nome do MPP (pouco legível), forma farmacêutica, concentração, via de administração, intervalo (incompleta, pouco legível/duvidoso e omissão) e abreviaturas (somente as pouco legíveis).

Os dados foram digitados, trabalhados em sua consistência, revistos e analisados no Epi Info 6.04. A análise exploratória dos dados foi feita por meio de estatística descritiva, com cálculo de medidas de posição (média, mediana) e variabilidade (desvio padrão e coeficiente de variação). Análises complementares foram realizadas no SPSS 11.0, a saber: regressão logística univariada, com cálculo de odds ratio (OR) para verificação da relação entre o tipo de prescrição e erros.

O presente estudo foi aprovado por Comitê de Ética em Pesquisa institucional em 30/01/2001.

\section{RESULTADOS}

Durante os 30 dias foram analisadas todas as prescrições contendo MPP recebidas na farmácia hospitalar, totalizando 4.026 prescrições de 456 pacientes (média de $8,8 \pm 0,4$ e mediana de 6,0 prescrições/paciente). $\mathrm{O}$ primeiro quartil apresentou um máximo de duas prescrições e o terceiro um mínimo de 13 , dos quais os últimos 5\% apresentaram mais de 28 prescrições. Quanto à estrutura da prescrição, destaca-se o predomínio daquelas escritas à mão. Houve erros no nome do paciente em $47,0 \%$ do total de prescrições e 19,3\% problemas de legibilidade (Tabela 1).

Quanto à identificação do profissional que elaborou a prescrição, observou-se uma relação de 1,3 prescritor por prescrição (5.153 registros para um total de 4.026 prescrições). Em 1.734 (33,7\%) dos registros houve dificuldades ou foi impossível identificar quem prescreveu.

Quando analisados os erros na prescrição dos MPP, verificou-se uma concentração em cinco tipos, envolvendo $93,5 \%$ dos registros, e $86,5 \%$ se refere a algum tipo de omissão de informação. Os erros relativos à concentração dos MPP somam 1.900 (59,8\% do total registrado) conforme Tabela 2 .

Assim, foram registrados 7.148 MPP em 4.026 prescrições, nas quais se observou algum tipo de erro em 3.177 (44,5\%). A prevalência de erros se apresentou significativamente diferenciada nos diversos setores. O centro de tratamento intensivo (CTI) e os setores de queimados agudos e crônicos apresentaram a maior relação de MPP por prescrição, enquanto o CTI, a unidade de tratamento intensivo (UTI), e os setores de neurologia e cuidados intermediários foram os que apresentaram maior percentual de MPP com erro 
Tabela 1. Características das prescrições analisadas: tipo de prescrição, nome do paciente, data e caligrafia. Minas Gerais, 2001. $(\mathrm{N}=4.026)$

\begin{tabular}{lccc}
\hline Característica & Classificação & $\mathrm{n}$ & $\%$ \\
\hline Tipo de & Escrita à mão & 1.840 & 45,7 \\
prescrição & Mista & 1.519 & 37,7 \\
Nome do & Pré-digitada & 667 & 16,6 \\
Paciente & Legível & 2.069 & 51,4 \\
& Incompleto & 1.329 & 33,0 \\
& Pouco legível/duvidoso & 480 & 11,9 \\
& Não identificado & 63 & 1,6 \\
Data & Alterado & 53 & 1,3 \\
& Ilegível & 32 & 0,8 \\
& Legível & 3.646 & 90,6 \\
& Pouco legível/duvidosa & 316 & 7,8 \\
& Omitida & 28 & 0,7 \\
& Incompleta & 30 & 0,7 \\
& Ilegível & 6 & 0,1 \\
& Louco legível/duvidosa & 743 & 18,5 \\
& Ilegível & 32 & 0,8 \\
\hline
\end{tabular}

(Figura). As proporções de erros por prescrição do CTI $(2,8)$ e cirurgia plástica $(0,2)$ mostraram diferença de 11,2 vezes na prevalência de erros entre os dois setores, na qual as clínicas de queimados agudos/crônicos e cirurgia plástica utilizavam predominantemente prescrições pré-digitadas e as demais usavam prescrição escrita à mão.
Tabela 2. Tipos e freqüências de erros nas prescrições de medicamentos potencialmente perigosos (MPP). Minas Gerais, 2001.

\begin{tabular}{lcccc}
\hline Tipo de erro & $\mathrm{n}$ & $\%$ & $(\%)^{\mathbf{a}}$ & $\begin{array}{c}\% \\
\text { acumulado }\end{array}$ \\
\hline $\begin{array}{l}\text { Concentração } \\
\quad \text { Omissão }\end{array}$ & $\begin{array}{c}1.581 \\
\quad 49,8\end{array}$ & 22,1 & 49,8 \\
$\quad \begin{array}{c}\text { Duvidosa } \\
\quad \text { Incompleta }\end{array}$ & 179 & 5,6 & 2,5 & 55,4 \\
$\begin{array}{l}\text { Omissão forma } \\
\text { farmacêutica }\end{array}$ & 807 & 25,4 & 11,3 & 85,2 \\
$\begin{array}{l}\text { Pouca legibilidade } \\
\text { do MPP }\end{array}$ & 264 & 8,3 & 3,7 & 93,5 \\
$\begin{array}{l}\text { Taxa de infusão } \\
\text { duvidosa }\end{array}$ & 47 & 1,5 & 0,7 & 95,0 \\
$\begin{array}{l}\text { Omissão via de } \\
\text { administração }\end{array}$ & 42 & 1,3 & 0,6 & 96,3 \\
Outros & 117 & 3,7 & 1,6 & 100,0 \\
\hline Total & 3.177 & 100,0 & 44,5 & \\
\hline
\end{tabular}

a Percentagem em relação ao número total de prescrições de MPP $(n=7.148)$.

A Tabela 3 mostra que $90 \%$ dos erros com MPP se concentraram em nove medicamentos, entre os quais a heparina, fentanil e midazolam. A heparina foi o MPP que originou mais erros, sendo mais freqüentes a omissão da forma farmacêutica e da concentração, a pouca legibilidade e a concentração incompleta. A heparina ampola e o midazolam foram responsáveis pela maioria dos erros relacionados à concentração. Verificou-se tendência à omissão da forma farmacêutica quando a heparina e o fentanil eram prescritos,

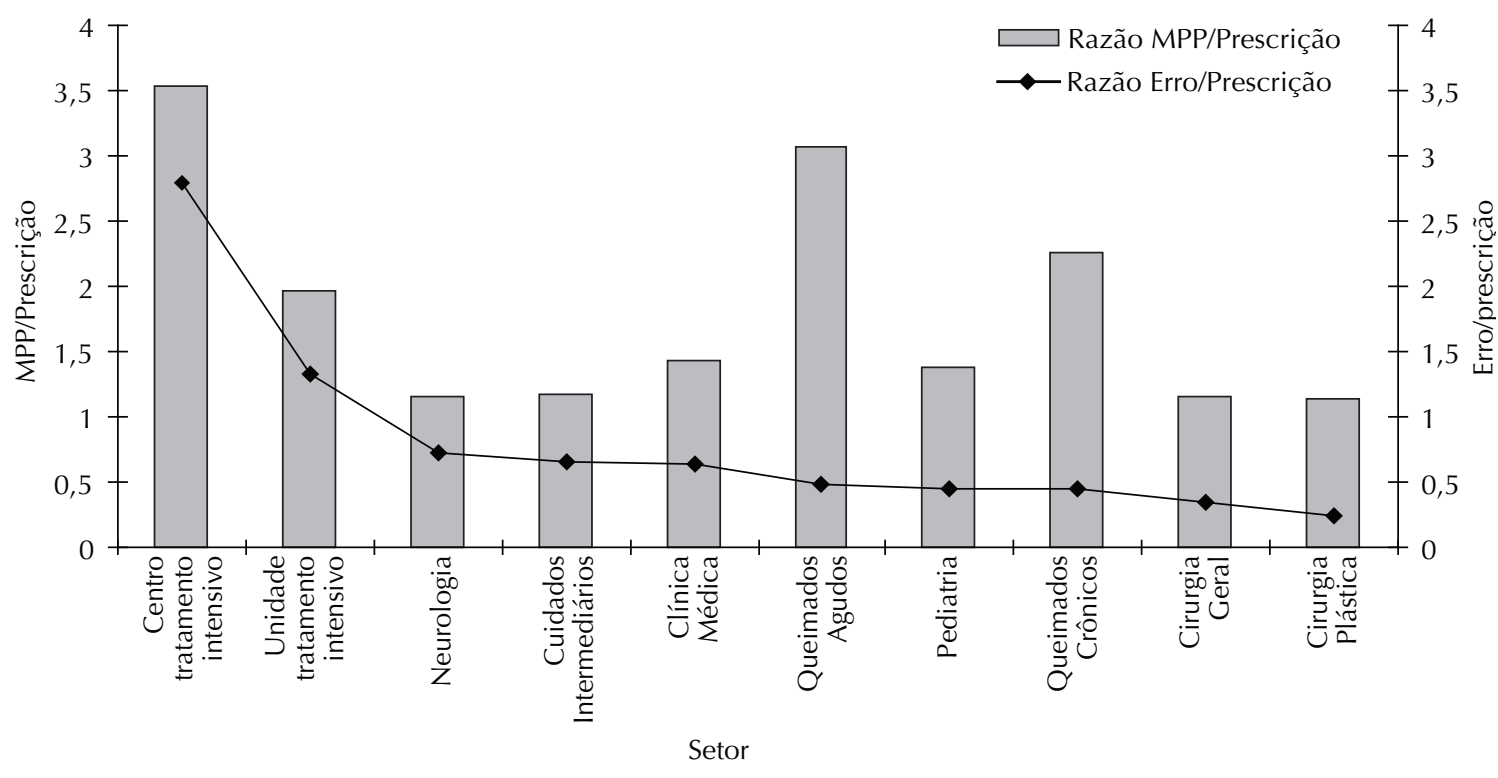

Figura. Número de erros e de medicamentos potencialmente perigosos (MPP) por prescrição e por setores em um hospital de referência. Minas Gerais, 2001. 
Tabela 3. Freqüência dos erros de prescrições por medicamento potencialmente perigoso. Minas Gerais, 2001.

\begin{tabular}{lccc}
\hline $\begin{array}{l}\text { Medicamento } \\
\text { potencialmente perigoso }\end{array}$ & $\mathrm{n}^{\mathrm{a}}$ & $\%$ & $\begin{array}{c}\% \\
\text { acumulado }\end{array}$ \\
\hline Heparina amp. 0,25mL & 1.072 & 33,7 & 33,7 \\
Fentanil & 669 & 21,1 & 54,8 \\
Midazolam & 363 & 11,4 & 66,3 \\
Nalbufina amp. 1mL & 305 & 9,6 & 75,8 \\
Pancurônio amp. 2mL & 143 & 4,5 & 80,3 \\
Dopamina amp. 20mL & 116 & 3,7 & 84,0 \\
Cloreto de potássio & 101 & 3,2 & 87,2 \\
amp.10mL & & & \\
Midazolam amp. 10mL & 58 & 1,8 & 89,0 \\
c/50mg & 48 & 1,5 & 90,5 \\
Tramadol fr. 10mL & 47 & 1,5 & 92,0 \\
Epinefrina amp. 2mL & 40 & 1,3 & 93,2 \\
Petidina amp. 2mL & 39 & 1,2 & 94,5 \\
Morfina amp. 1mL & 176 & 5,5 & 100,0 \\
Outros & 3.177 & 100,0 & \\
\hline Total & & & \\
\hline
\end{tabular}

a Número de erros registrados no total de prescrições analisadas.

Tabela 4. Relação entre o tipo de prescrição e a freqüência de erros. Minas Gerais, 2001.

\begin{tabular}{lccc}
\hline Tipo de prescrição & OR & IC 95\% & $\mathrm{p}$ \\
\hline Pré-digitada & - & - & 0,00 \\
Escrita à mão & 2,96 & 2,$47 ; 3,55$ & 0,00 \\
Mista & 2,49 & 2,$07 ; 3,00$ & 0,00 \\
\hline
\end{tabular}

pois juntos, originaram a quase totalidade dos registros assim classificados. Para o fentanil os erros mais freqüentes foram omissão da forma farmacêutica, da concentração, da via da administração e medicamento pouco legível e para o midazolam os problemas estavam relacionados principalmente à omissão da concentração, concentração duvidosa e medicamento pouco legível. Ressaltam-se também os erros observados na prescrição do cloreto de potássio injetável: taxa de infusão duvidosa, medicamento pouco legível, concentração duvidosa, falta via de administração, concentração incompleta, omissão da concentração e via de administração pouco legível/duvidosa.

Foram observados 23 erros de prescrição do tipo decisão, um sendo de forma farmacêutica, dez de concentração e 12 de administração. O MPP mais prevalente foi a heparina, com 43,5\% dos registros. Foram computados somente os erros de decisão onde foi possível identificá-los a partir da prescrição.

Nas 4.026 prescrições foram registrados 70 tipos diferentes de abreviaturas, com um total de 133.956 ocorrências (média de 33,3 por prescrição). Desse total, 5.427 foram classificadas como pouco legíveis. As abreviaturas mg (miligrama), h (hora), mL (mililitro), cp/comp/cp (comprimido), EV/IV (endovenoso/ intravenoso), gts (gotas), amp (ampola), vo (via oral), $\mathrm{g} / \mathrm{gr}$ (grama), SF (soro fisiológico), $\mathrm{min} / \mathrm{X}^{\prime}$ (minuto), sc (sub-cutâneo), ABD (água bidestilada), S/N e SN (se necessário) e ACM (a critério médico) representavam $90 \%$ delas. O maior número de abreviaturas observado em uma única prescrição foi de 211. As abreviaturas UI ou $U$ foram utilizadas 2.062 vezes em 1.971 prescrições (48,9\% do total de prescrições).

O total de erros de prescrição (redação e decisão) foi de 13.387, com uma média de 3,3 por prescrição, sendo $99,8 \%$ dos registros de erros de redação. Os erros de redação somaram 13.364 eventos classificados em: 3.154 (erros com MPP), 1.894 (nome do paciente), 380 (data da prescrição), 775 (prescrição pouco legível ou ilegível), 5.427 (abreviaturas pouco legíveis) e 1.734 (identificação do prescritor).

A análise de regressão logística univariada, mostrada na Tabela 4, revela que o tipo de prescrição tem influência nos erros de prescrição (decisão e redação). Foi testado o tipo de prescrição: pré-digitada, escrita à mão e mista, e considerada a prescrição pré-digitada como referência para essa comparação. Observou-se que a chance de erros na prescrição escrita à mão foi aproximadamente três vezes mais freqüente que na pré-digitada e na prescrição mista esta chance de erros foi 2,5 vezes mais freqüente.

\section{DISCUSSÃO}

A presente investigação corrobora indicações internacionais da importância dos erros de prescrição de MPP em ambientes hospitalares, seja do ponto de vista de sua prevalência ou de seu potencial de risco aos pacientes. ${ }^{2,8,14}$ Entretanto, algumas limitações devem ser ressaltadas: uma diz respeito à probabilidade de falhas no diagnóstico dos erros de decisão, pois a análise isolada de prescrições, sem considerar a condição clínica do paciente e sem discutir o caso com o prescritor, não permite avaliar alguns tipos de erros. Por esta razão, somente foram classificados como erros de decisão: forma farmacêutica, concentração, via de administração, intervalo e taxa de infusão errados. Assim, erros de decisão podem não ter sido registrados, provocando subestimação nos dados.

Outra limitação relevante refere-se à subjetividade da avaliação da legibilidade. Para seu controle foram realizadas avaliações independentes por profissionais com larga experiência na leitura de prescrições, decisão centralizada em um profissional com experiência maior e consenso entre eles em caso de dúvidas. 
A terceira limitação é que foi testada somente uma variável para explicar a ocorrência de erros de prescrição. Outros fatores podem ter influído na prevalência encontrada de erros de prescrição, mas não foram considerados no presente estudo, cujo interesse foi o tipo de emissão da prescrição.

Problemas de identificação do paciente, do prescritor e na data apareceram em $47,0 \%, 33,7 \%$ e em $9,4 \%$ das prescrições, respectivamente, situações que aumentam a probabilidade de erros. Miasso \& Cassiani ${ }^{18}$ (2000) verificaram que $33,9 \%$ dos erros de administração de medicamentos em um hospital de ensino foram os problemas de identificação do paciente. Como documento legal, a prescrição deve ter a identificação não apenas do paciente, mas também do emitente, para que em situações de esclarecimento de dúvidas, seja possível localizar o responsável pela prescrição. E, em um ambiente hospitalar, onde centenas ou mesmo milhares de doses de medicamentos são dispensadas diariamente pela farmácia hospitalar, a omissão da data do documento pode também levar a erros.

A diferença de ocorrência de erros na prescrição de MPP entre os setores (Figura) guarda uma relação inversa com a utilização da prescrição pré-digitada. Esta relação reforça o papel do tipo de prescrição na determinação dos erros e pode ser explicada em parte pelo tipo de prescrição adotada nos variados setores de internação.

A heparina, ao longo dos anos, tem sido associada a altas taxas de erros e está entre os dez medicamentos mais freqüentemente relatados em notificações de erros com danos provocados em pacientes nos Estados Unidos. Entre 1999 e 2002, a heparina ocupou os primeiros lugares nos registros de erros graves $(4,5 \%$ a $5,5 \%$ ) e, em 2002, foi responsável por $9,5 \%$ dos erros que causaram lesões nos pacientes. ${ }^{19}$

Nas prescrições do fentanil, o segundo medicamento mais envolvido em erros $(21,1 \%)$, a omissão da forma farmacêutica foi o erro mais freqüente, uma falha que pode acarretar a troca de uma apresentação pela outra. O fentanil é um derivado opióide de grande potência e seu uso indevido pode acarretar problemas graves para o paciente. A prescrição do midazolam com a omissão da concentração ou concentração duvidosa é também relevante por ser utilizado com freqüência em terapia intensiva, sendo fundamental a administração da dose correta.

Os erros na prescrição do cloreto de potássio injetável ressaltam a necessidade de prevenção de acidentes graves. Uma infusão ou diluição errada deste medicamento, ou administração do produto concentrado, pode ter conseqüências fatais. ${ }^{2,8,13}$ Em estudo realizado em 24 unidades de terapia intensiva no Reino Unido, com análise de 21.589 prescrições, os cinco medicamentos mais envolvidos em prescrições incorretas foram: cloreto de potássio (10,2\%), heparina $(5,3 \%)$, sulfato de magnésio $(5,2 \%)$, paracetamol $(3,2 \%)$ e propofol $(3,1 \%) .^{20}$

A falta de padronização e o freqüente uso de abreviaturas (33,3/prescrição) mostram falhas latentes que podem contribuir para a ocorrência de erros de medicação. O uso intensivo da abreviatura UI ou I pode levar a erros graves, pela possibilidade de ser confundida como o número zero e acarretar administração de concentração 10 ou 100 vezes maior do que a prescrita. ${ }^{1,2}$ A utilização de abreviaturas em prescrições médicas está entre as causas mais citadas de erros de medicação por seu potencial de confusão e falhas de comunicação, sendo já antiga a idéia de eliminar o seu uso. O Institute for Safe Medication Practices (ISMP) tem se preocupado com este problema há vários anos. A Joint Commission on Accreditation of Healthcare Organizations, instituição que acredita hospitais no mundo todo, estabeleceu a proibição do uso de uma lista de abreviaturas, dentre elas o U e UI, nos hospitais candidatos a acreditação. ${ }^{1}$ Segundo Koczmara et al $^{11}$ (2005), alguns nomes nunca devem ser abreviados devido aos freqüentes enganos provocados, estando entre eles: unidades, microgramas, sub-cutâneo e centímetro cúbico.

Um exemplo clássico de falha na utilização da abreviatura UI foi registrado no Canadá, onde ocorreu erro de administração por equívoco na dose da insulina. $\mathrm{O}$ paciente teve lesão grave permanente, pois recebeu 70 unidades de insulina em vez das sete prescritas, porque a abreviatura $U$ foi confundida com o número zero. ${ }^{11}$

A alta prevalência dos erros de redação em ambiente hospitalar também foi observada por Lisby et $\mathrm{al}^{15}$ (2005). Os autores verificaram que $75 \%$ dos erros encontrados nas prescrições podem ser classificados como erros de redação, sendo os mais prevalentes os problemas com a forma farmacêutica, a omissão da dose e a via de administração. Em outro estudo, realizado em clínicas médicas de 60 unidades de saúde nos Estados Unidos, foi observado que os erros não clínicos, ou de redação, foram responsáveis por $79,1 \%$ dos registros. ${ }^{7}$

Os erros de redação levantados na presente pesquisa e relacionados aos MPP poderão ser minimizados com a padronização e elaboração de normas para a prescrição de medicamentos. , $17,22^{A} \mathrm{~A}$ ausência de padrão definido e o intenso uso de abreviaturas nas prescrições estudadas, como UI significando unidades, enfatizam a necessidade de padronização do uso de abreviaturas nas prescrições. Sugere-se que algumas abreviaturas como a UI sejam eliminadas das prescrições devido ao potencial de erros associado. ${ }^{1}$

Quanto aos problemas de legibilidade, uma importante diminuição poderá ser obtida com a padronização do tipo de prescrição a ser utilizada em ambiente hospitalar. Erros de prescrição podem ser reduzidos com a 
utilização de editores de textos para a prescrição ou prescrições pré-digitadas, evitando sempre que possível as prescrições feitas à mão. ${ }^{22}$

Uma abordagem diferente deve ser utilizada para a redução dos erros de prescrição do tipo decisão, já que medidas de aperfeiçoamento do conhecimento do prescritor e interceptação desses tipos de erros são diferentes daquelas preconizadas para erros de redação. ${ }^{2}$ A importância de problemas de decisão na prescrição foi observada em estudo sobre antibióticos realizado em hospital universitário brasileiro, tendo sido registrados 91 incidentes, dos quais $7,7 \%$ foram considerados erros de prescrição e de tipo de decisão. ${ }^{16}$

Conforme Aspden et $\mathrm{al}^{2}$ (2007), as recomendações com maior evidência científica para a prevenção de erros de medicação em hospitais são: adoção da prescrição eletrônica com o devido suporte clínico, inclusão de farmacêuticos nas visitas clínicas, viabilização de contato com farmacêuticos durante 24 horas para solucionar dúvidas em relação a medicamentos, e presença de procedimentos especiais e protocolos escritos para o uso de MPP. Esta última medida poderá prevenir parte considerável dos erros com medicamentos de alto risco..$^{2,8,13}$ Entretanto, pesquisas devem ser feitas em hospitais brasileiros para verificar o real impacto da implantação de protocolos no uso dos MPP e na prevenção de eventos relacionados a esses medicamentos em nossa realidade.

A implantação da prescrição eletrônica pode ter forte impacto nos erros de prescrição, devendo ser buscada a sua instituição. Dado que o seu custo pode ser impeditivo para parte dos hospitais brasileiros, é recomendável a adoção de prescrição pré-digitada ou editada para evitar ao máximo as prescrições escritas à mão. ${ }^{2}$ Entretanto, há necessidade da elaboração cuidadosa das prescrições pré-digitadas ou utilização de editores de texto para prescrição, no sentido de evitar o aparecimento de novos tipos de erros ou a simples transposição de antigos problemas para um novo modo de prescrever.

Finalmente, observa-se nos achados da presente investigação e no seu confronto com a literatura internacional, que os erros de medicação envolvendo os MPP tendem a apresentar padrões definidos, fato importante para a tomada de decisões dirigidas ao seu controle. Todavia, todo conhecimento dessa natureza, quando produzido em ambiente diverso, implica a necessidade de adaptação à realidade cultural onde se objetiva intervir e ao perfil dos problemas detectados em cada local. 


\section{REFERÊNCIAS}

1. Abushaiqa ME, Zaran FK, Bach DS, Smolarek RT, Farber MS. Educational interventions to reduce use of unsafe abbreviations. Am J Health Syst Pharm 2007;64(11):1170-3. DOI: 10.2146/ajhp060173

2. Aspden P, Wolcott J, Bootman JL, Cronenwett LR, Committee on Identifying and Preventing Medication Errors. Preventing medication errors. Quality Chasm Series (Hardcover). Washington: National Academies Press; 2007

3. Barber N, Rawlins M, Dean Franklin B. Reducing prescribing error: competence, control, and culture. Qual Saf Health Care. 2003;12(Suppl 1):i29-32. DOI: 10.1136/qhc.12.suppl_1.i29

4. Brennan TA, Leape LL, Laird NM, Hebert L, Localio AR, Lawthers AG, et al. Incidence of adverse events and negligence in hospitalized patients: results of the Harvard Medical Practice Study I. N Engl / Med. 1991;324(6):370-6.

5. Cohen MR, Proulx SM, Crawford, SY. Survey of hospital systems and common serious medication errors. J Healthc Risk Manag. 1998;18(1):16-27.

6. Dean B, Barber N, Schachter V. What is prescribing error? Qual Health Care. 2000;9(4):232-7. DOI: 10.1136/qhc.9.4.232

7. Devine EB, Wilson-Norton JL, Lawless NM, Hansen RN, Hazlet TK, Kelly K, et al. Characterization of prescribing errors in an internal medicine clinic. $A m$ J Health Syst Pharm. 2007;64(10):1062-70. DOI: 10.2146/ajhp060125

8. Federico F. Preventing harm from high-alert medications. It Comm J Qual Patient Saf. 2007;33(9):537-42.

9. Fuchs FD, Wannmacher L. Farmacologia clínica: fundamentos da terapêutica racional. Rio de Janeiro: Guanabara Koogan; 1998.

10. Kohn LT, Corrigan JM, Donaldson MS. To err is human: building a safer system. Washington: National Academy Press; 1999.

11. Koczmara C, Jelincic V, Dueck C. Dangerous abbreviations: " $U$ " can make a difference! Dynamics. 2005;16(3):11-5

12. Leape LL, Brennan TA, Laird N, Lawthers AG, Localio AR, Barnes BA, et al. The nature of adverse events and negligence in hospitalized patients. Results of the Harvard Medical Practice Study II. N Engl / Med. 1991;324(6):377-84.
13. Leape LL, Berwick DM, Bates DW. What practices will most improve safety? JAMA. 2002;288(4):501-7. DOI: 10.1001/jama.288.4.501

14. Leape LL, Berwick DM. Five years to err is human. What have we learned? JAMA. 2005;293(19):2384-90. DOI: 10.1001/jama.293.19.2384

15. Lisby M, Nielsen LP, Mainz J. Errors in the medication process: frequency, type, and potential. Int I Qual Health Care. 2005;17(1):15-22. DOI: 10.1093/intghc/ mzi015

16. Louro E, Romano-Lieber NS, Ribeiro E. Eventos adversos a antibióticos em pacientes internados em um hospital universitário. Rev Saude Publica. 2007; 41(6):1042-8.

17. Meyer TA. Improving the quality of the order-writing process for inpatient orders and outpatient patients. Am J Health Syst Pharm. 2000;57(Suppl 4):S18-S22.

18. Miasso Al, Cassiani SHB Erros na administração de medicamentos: divulgação de conhecimentos e identificação do paciente como aspectos relevantes. Rev ESC Enferm USP. 2000;34(1):16-25.

19. Niccolai CS, Hicks RW, Oertel L, Francis JL. Heparin Consensus Group. Unfractionated heparin: focus on a high-alert drug. Pharmacotherapy. 2004;24(8 Pt 2):146S-155S. DOI: 10.1592/phco.24.12.146S.36107

20. Ridley SA, Booth SA, Thompson CM, Intensive Care Society's Working Group on Adverse Incidents. Prescription errors in UK critical care units. Anaesthesia. 2004;59(12):1193-200. DOI: 10.1111/ j.1365-2044.2004.03969.x

21. Rosa MB, Perini E. Erros de medicação: quem foi? Rev Assoc Med Bras. 2003;49(3):335-41. DOI: 10.1590/ S0104-42302003000300041

22. Tran M. The impact of introducing pré-printed chemotherapy medication charts to a day chemotherapy unit. J Oncol Pharm Pract. 2000;6(2):64-9. DOI: 10.1177/107815520000600206

23. World Health Organization. World alliance for patient safety: forward programme 2006-2007. Geneva; 2006 [citado 2007 dez 29]. Disponível em: http://www.who. int/patientsafety/en

24. Yu KH, Nation RL, Dooley MJ. Multiplicity of medication safety terms, definitions and functional meanings: when is enough enough? Qual Saf Health Care. 2005;14(5):358-63. DOI: 10.1136/ qshc.2005.014159

Artigo baseado na dissertação de mestrado de Rosa MB, apresentada à Universidade Federal de Minas Gerais, Escola de Veterinária, em 2002.

Pesquisa parcialmente financiada pela Fundação Hospitalar do Estado de Minas Gerais. 